\title{
Targeting of nonlipidated, aggregated apoE with antibodies inhibits amyloid accumulation
}

\author{
Fan Liao, ${ }^{1}$ Aimin Li, ${ }^{1}$ Monica Xiong, ${ }^{1}$ Nga Bien-Ly, ${ }^{2}$ Hong Jiang, ${ }^{1}$ Yin Zhang, ${ }^{2}$ Mary Beth Finn, ${ }^{1}$ Rosa Hoyle, ${ }^{1}$ Jennifer Keyser, ${ }^{1}$ \\ Katheryn B. Lefton, ${ }^{1}$ Grace O. Robinson, ${ }^{1}$ Javier Remolina Serrano, ${ }^{1}$ Adam P. Silverman, ${ }^{2}$ Jing L. Guo, ${ }^{2}$ Jennifer Getz, ${ }^{2}$ Kirk Henne, ${ }^{2}$ \\ Cheryl E.G. Leyns, ${ }^{1}$ Gilbert Gallardo, Jason D. Ulrich, ${ }^{1}$ Patrick M. Sullivan, ${ }^{3}$ Eli Paul Lerner, ${ }^{4}$ Eloise Hudry, ${ }^{4}$ Zachary K. Sweeney, ${ }^{2}$ \\ Mark S. Dennis, ${ }^{2}$ Bradley T. Hyman, ${ }^{4}$ Ryan J. Watts, ${ }^{2}$ and David M. Holtzman ${ }^{1}$ \\ 'Department of Neurology, Hope Center for Neurological Disorders, Charles F. and Joanne Knight Alzheimer's Disease Research Center, Washington University School of Medicine, St. Louis, Missouri, \\ USA. ${ }^{2}$ Denali Therapeutics Inc., South San Francisco, California, USA. ${ }^{3}$ Department of Medicine, Duke University, Durham, North Carolina, USA. ${ }^{4}$ MassCeneral Institute for Neurodegenerative Disease, \\ Massachusetts General Hospital and Harvard Medical School, Charlestown, Massachusetts, USA.
}

\begin{abstract}
The apolipoprotein E E4 allele of the APOE gene is the strongest genetic factor for late-onset Alzheimer disease (LOAD). There is compelling evidence that apoE influences Alzheimer disease (AD) in large part by affecting amyloid $\beta$ (A $\beta$ ) aggregation and clearance; however, the molecular mechanism underlying these findings remains largely unknown. Herein, we tested whether anti-human apoE antibodies can decrease $A \beta$ pathology in mice producing both human $A \beta$ and apoE4, and investigated the mechanism underlying these effects. We utilized APPPS1-21 mice crossed to apoE4-knockin mice expressing human apoE4 (APPPS1-21/APOE4). We discovered an anti-human apoE antibody, anti-human apoE 4 (HAE-4), that specifically recognizes human apoE4 and apoE3 and preferentially binds nonlipidated, aggregated apoE over the lipidated apoE found in circulation. HAE-4 also binds to apoE in amyloid plaques in unfixed brain sections and in living APPPS1-21/APOE4 mice. When delivered centrally or by peripheral injection, HAE-4 reduced A $\beta$ deposition in APPPS1-21/APOE4 mice. Using adeno-associated virus to express 2 different full-length anti-apoE antibodies in the brain, we found that HAE antibodies decreased amyloid accumulation, which was dependent on Fcy receptor function. These data support the hypothesis that a primary mechanism for apoE-mediated plaque formation may be a result of apoE aggregation, as preferentially targeting apoE aggregates with therapeutic antibodies reduces $A \beta$ pathology and may represent a selective approach to treat $A D$.
\end{abstract}

\section{Introduction}

Alzheimer disease (AD) is the most common form of dementia and it affects more than 5 million people in the United States (www.alz. org). Compelling evidence has shown that amyloid $\beta(\mathrm{A} \beta)$ plays a key role in the pathogenesis of $\mathrm{AD}(1,2)$. In autosomal dominant $\mathrm{AD}$, missense mutations in amyloid precursor protein (APP) or components of the $\gamma$ - secretase complex presenilin 1 (PS1) or presenilin 2 (PS2) result in early onset amyloid deposition in the brain due to a relative increase in production of longer $A \beta$ species such as $A \beta_{42}$, an increase in all $A \beta$ species, or an alteration of $A \beta$ aggregation propensity and clearance (3). Also, a protective mutation in $\mathrm{AD}$ was recently described near the $\mathrm{N}$-terminus of the $\beta$ secretase site in APP that results in decreased $A \beta$ production (4).

\section{Related Commentary: p. 1734}

\section{Authorship note: $\mathrm{FL}$ and $\mathrm{AL}$ contributed equally to this work.}

Conflict of interest: NBL, YZ, APS, JLG, JG, KH, ZS, MSD, and RW are employees of Denali. $\mathrm{FL}, \mathrm{HJ}$, and $\mathrm{DMH}$ are inventors on a patent filed by Washington University on the topic of anti-apoE antibodies that was licensed by Denali. DMH cofounded and is on the scientific advisory board of C2N Diagnostics. DMH consults for Genentech, AbbVie, Eli Lilly, Proclara, and Denali. Washington University receives research grants to the lab of DMH from C2N Diagnostics, Eli Lilly, AbbVie, and Denali.

Submitted: July 20, 2017; Accepted: February 1, 2018.

Reference information: / Clin Invest. 2018;128(5):2144-2155.

https://doi.org/10.1172/JCI96429.
The majority of AD cases are known as late-onset AD (LOAD), which clinically begins after the age of 65 . The apolipoprotein $\mathrm{E}$ (APOE) gene is the strongest genetic risk factor for LOAD. In humans, the 3 common isoforms of apoE $-E 2, E 3$, and $E 4-$ are encoded by a 299 amino acid protein. Compared with the most common form of apoE, apoE3 (Cys112, Arg158), each allele of apoE4 (Arg112, Arg158) strongly increase the risk for AD ( 3.7-fold for one $E 4$ allele and $~ 12$-fold for $2 E 4$ alleles relative to the E3/ E3 genotype); in contrast, apoE2 (Cys112, Cys158) is protective for $\mathrm{AD}(5,6)$. How apoE impacts $\mathrm{AD}$ pathogenesis is not entirely clear; however, evidence shows that apoE influences $\mathrm{A} \beta$ aggregation and clearance $(7)$ and is also found in amyloid plaques $(8,9)$.

Previously, our group has shown that passive immunotherapy using HJ6.3, an antibody targeting endogenous murine apoE, strongly suppresses A $\beta$ pathology in the APPswe/PS1 $\triangle \mathrm{E} 9$ mouse brain when treatment is started prior to plaque onset (10). When administered after plaque onset, HJ6.3 reduced brain $A \beta$ plaque load, restored resting-state functional connectivity, and mildly improved spatial performance in the water maze (11). However, in order to further explore whether such an approach could potentially translate into an immunotherapy for humans, studies with apoE antibodies targeting human apoE in animals expressing human $\mathrm{A} \beta$ and apoE are critical. It is also important to understand the mechanism(s) underlying the therapeutic effects. 
A
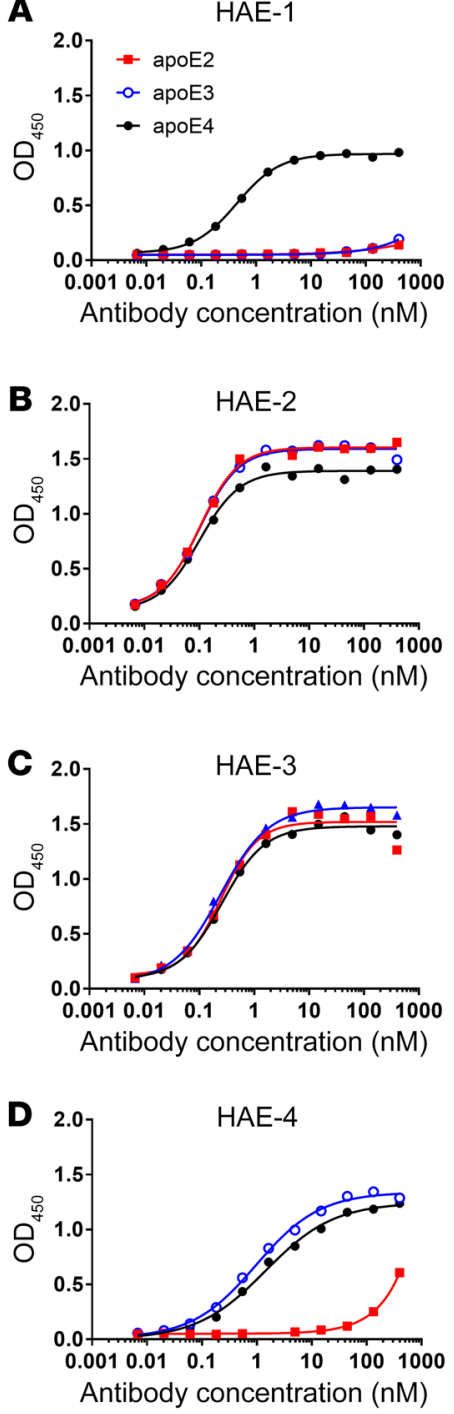
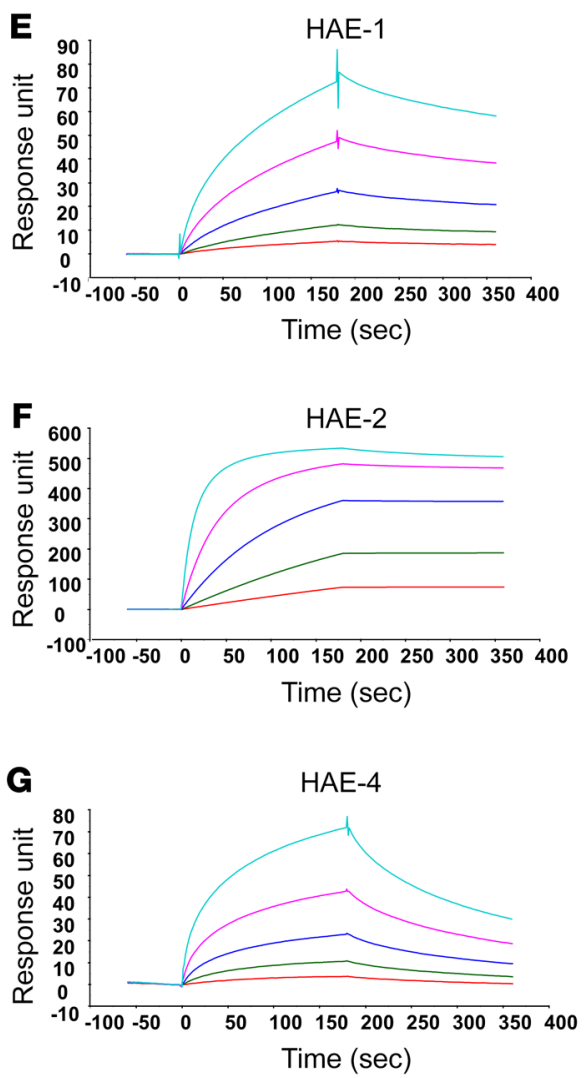

Figure 1. Characterization of HAE series anti-apoE antibodies. (A-D) Antibody binding to plates coated with recombinant apoE2, apoE3, or apoE4. Serially titrated $\mathrm{HAE}$ antibodies were incubated and binding was detected with HRP anti-mouse IgG antibody. (E-C) SPR was used to detect 3-fold serially diluted apoE antibody (starting at $100 \mathrm{nM}$ for HAE-2 and 1000 $\mathrm{nM}$ for HAE-1 and HAE-4) binding to biotinylated recombinant apoE4 captured on a streptavidin chip. Samples were injected at a flow rate of $30 \mu \mathrm{l} / \mathrm{min}$.

no appreciable binding to apoE2 (Figure 1D). HAE-1 was apoE4-specific (Figure 1A). Using surface plasmon resonance (SPR) (Figure 1, $\mathrm{E}-\mathrm{G})$, the apparent $\mathrm{K}_{D}$ values were determined for HAE-1 $\left(6.02 \times 10^{-8} \mathrm{M}\right)$, HAE-2 $\left(3.19 \times 10^{-10}\right.$ $\mathrm{M})$, and HAE-4 $\left(1.98 \times 10^{-7} \mathrm{M}\right)$ for their interaction with recombinant apoE4 (Table 1). The $K_{D}$ value for HAE-3 was not determined because it showed binding characteristics similar to those of HAE-2 in ELISA (Figure 1, $\mathrm{B}$ and $\mathrm{C}$ ). Oddly, the maximum response units for HAE-1 and HAE-4 as measured by SPR were only $10 \%$ of the maximum value seen for HAE-2, despite greater apoE 4 capture levels for experiments with HAE- 1 and HAE-4. This suggested the possibility that these 2 antibodies were recognizing a minor fraction of the immobilized apoE, a hypothesis that was later validated (see below).

Effects of intracerebroventricularly administered anti-apoE antibodies on $A \beta$ pathology in APPPS1-21/APOE4 mice. apoE is present in $\mathrm{A} \beta$-containing amyloid plaques in both human $\mathrm{AD}$ as well as in mouse models of amyloidosis $(8,9,14)$. To test whether HAE antibodies are

In the present study, we tested the effects of anti-human apoE (HAE) antibodies, a new series of apoE antibodies targeting human apoE, on A $\beta$ pathology in APPPS1-21/APOE4-knockin (KI) mice. These mice were generated by crossing human apoE4-KI mice expressing human apoE 4 under the control of endogenous murine apoE regulatory elements (APOE4) (12) to line APPPS1-21 (13). We found that HAE-4, an antibody specific for apoE3 and apoE4, was able to reduce $A \beta$ deposition when infused directly into the brain, delivered by i.p. injection starting at the time of plaque onset, or expressed in the brain via adeno-associated virus-mediated (AAVmediated) delivery. Here we present in detail the features of antibody HAE- 4 that facilitate its ability to decrease $\mathrm{A} \beta$ deposition and propose a likely mechanism of action.

\section{Results}

Characterization of the HAE series of apoE antibodies using recombinant human apoE. We used a direct ELISA to test whether the HAE series of apoE antibodies binds to different recombinant apoE isoforms (Figure 1, A-D). HAE-2 and HAE-3 recognized all 3 isoforms of human apoE (Figure 1, B and $\mathrm{C}$ ), whereas HAE-4 had much higher affinity for apoE3 and apoE4 with able to decrease A $\beta$ pathology in APPPS1-21/APOE4 mice, we directly infused the antibodies into the lateral ventricle of the mice before plaque onset, starting at the age of 2 months. Antibodies were infused continuously $(0.3 \mu \mathrm{g} / \mathrm{h})$ via an osmotic pump for 6 weeks and mice were assessed at the age of 3.5 months (females, $n=10-11$ per group). Infusions of PBS or a control mouse IgG2ab into the mouse brain were included as negative controls, and anti$\mathrm{A} \beta$ antibody HJ5.1 (15) was used as a positive control. A $\beta$ plaques were stained using anti-A $\beta$ antibody HJ3.4 (Supplemental Figure 1A; supplemental material available online with this article; Table 1. Apparent $K_{D}$ values of HAE-1, HAE-2, and HAE-4
calculated based on the SPR experiment

$\begin{array}{lccc}\text { Antibody } & \text { Ka (1/Ms) } & \text { Kd (1/s) } & \text { Apparent } K_{D}(M) \\ \text { HAE-1 } & 2.0 \mathrm{E}+04 & 1.2 \mathrm{E}-03 & 6.0 \mathrm{E}-08 \\ \text { HAE-2 } & 6.6 \mathrm{E}+05 & 2.1 \mathrm{E}-04 & 3.2 \mathrm{E}-10 \\ \text { HAE-4 } & 2.6 \mathrm{E}+04 & 5.2 \mathrm{E}-03 & 2.0 \mathrm{E}-07\end{array}$


A
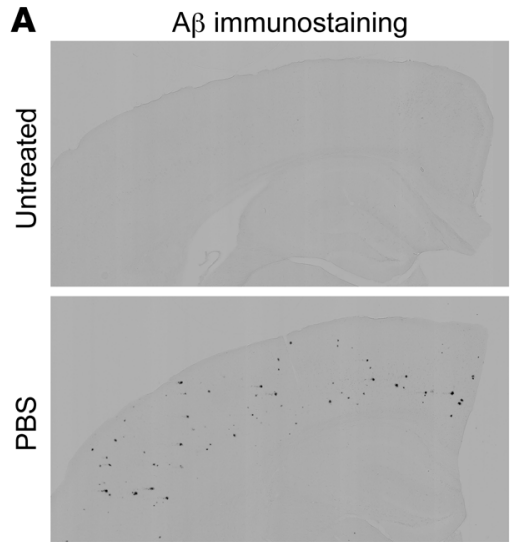

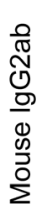

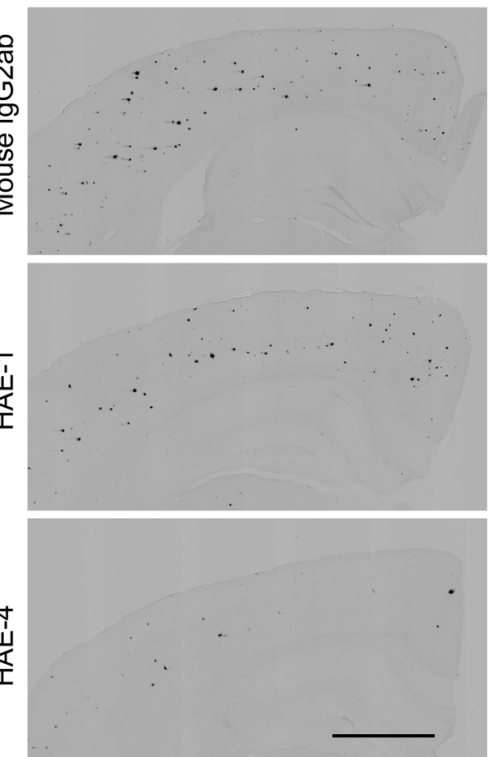

X-34
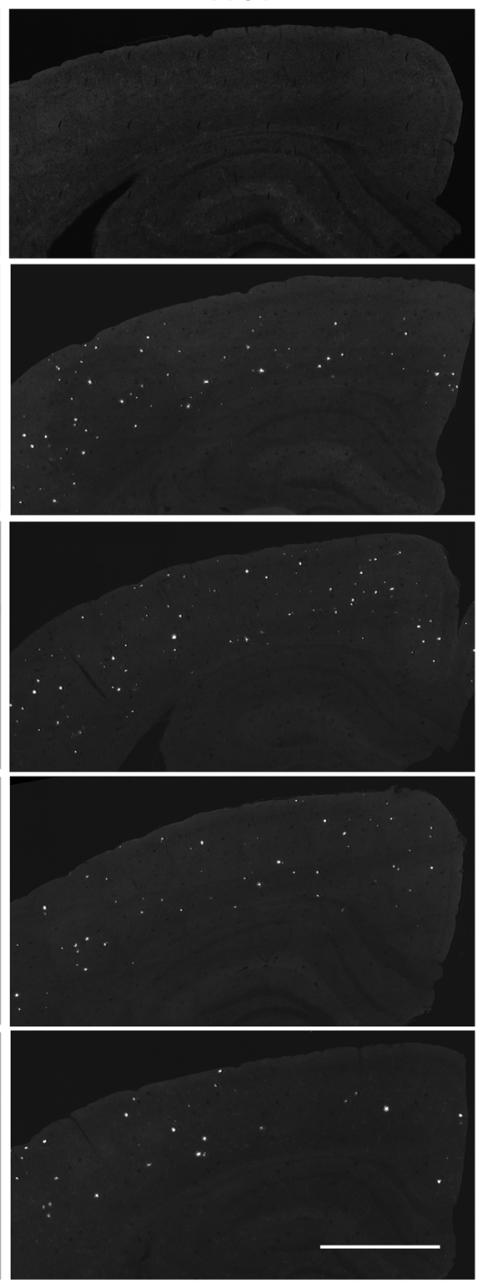

B

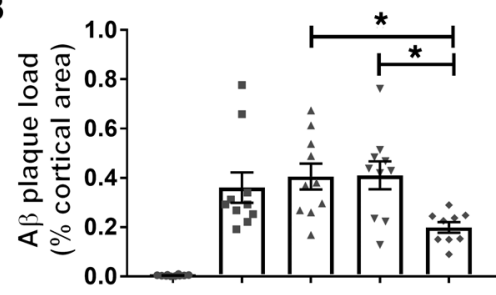

C

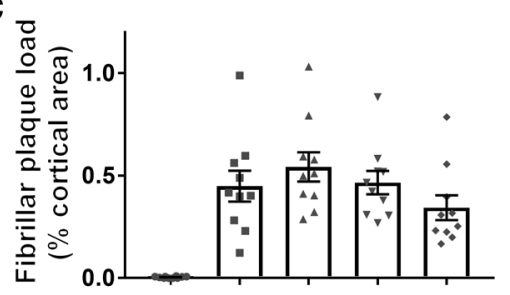

D

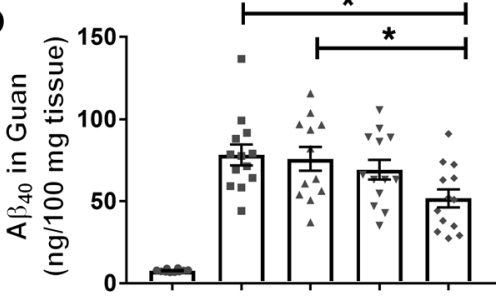

E

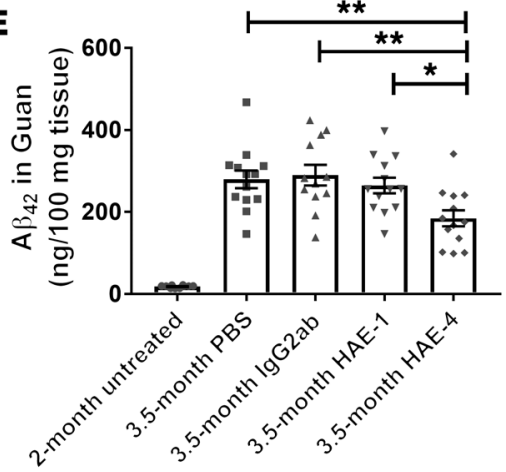

Figure 2. Effects of intraperitoneally administered anti-apoE antibodies on amyloid pathology in APPPS1-21/APOE4 mice. At the age of 2 months, the mice were injected i.p. weekly with $50 \mathrm{mg} / \mathrm{kg}$ antibodies (all female, $n=10-13 /$ group). The mice were sacrificed at the age of 3.5 months and the $A \beta$ pathology in the brain was assessed using histology and biochemical approaches. (A) Representative A $\beta$ staining using HJ3.4 (left panel) and representative X-34 staining for fibrillar plaques (right panel). Scale bar $=1 \mathrm{~mm}$. (B) Quantification of HJ3.4 staining. (C) Quantification of X-34 staining. (D) A $\beta_{40}$ and $(E) A \beta_{42}$ was measured by ELISA in insoluble fractions of tissue lysates (Guan = Guanidine) by ELISA. One-way ANOVA followed by Tukey's $t$ test was performed to compare different groups shown in B-E. Data are mean $\pm \mathrm{SEM} .{ }^{*} P<0.05,{ }^{*} P<0.01$.

https://doi.org/10.1172/JCI96429DS1), and fibrillar plaques were stained using Thioflavin S (Supplemental Figure 1B). The cortical tissue was extracted sequentially in PBS (soluble), $1 \%$ Triton $\mathrm{X}-100$, and $5 \mathrm{M}$ guanidine $\mathrm{HCl}$ (insoluble). Levels of insoluble $\mathrm{A} \beta_{40}$ and $\mathrm{A} \beta_{42}$ were determined by ELISA (Supplemental Figure 1, $\mathrm{C}$ and D). HJ5.1 (anti-A $\beta$ ) and the anti-apoE antibodies HAE-1 and HAE-4 significantly reduced $A \beta$ plaque load compared with a control mouse IgG2ab (Supplemental Figure 1A). HAE-4 also significantly reduced A $\beta$ plaque load compared with PBS (Supplemental Figure 1A). Some of the antibodies tended to decrease fibrillar $A \beta$ and insoluble $A \beta_{40}$ and $A \beta_{42}$, but the effects were not statistically significant (Supplemental Figure 1, B-D).

Effects of peripheral administration of anti-apoE antibodies on $A \beta$ pathology in APPPS1-21/APOE4 mice. Because i.c.v.-administered $\mathrm{HAE}-1$ and $\mathrm{HAE}-4$ significantly reduced the $\mathrm{A} \beta$ plaque load in APPPS1-21/APOE4 mice, we tested whether peripheral administration of HAE- 1 and HAE- 4 could impact $A \beta$ pathology in these mice. Beginning at the age of 2 months, APPPS1-21/ APOE 4 mice (females, $n=10-13$ per group) were treated weekly with i.p. injections ( $50 \mathrm{mg} / \mathrm{kg}$ body weight) of antibodies until 3.5 months of age. A group of untreated mice were harvested at 2 months to determine baseline $A \beta$ pathology. As determined by $A \beta$ immunostaining, HAE- 4 significantly reduced $A \beta$ plaque load compared with the mouse IgG2ab control (Figure 2, A and B). X-34 staining suggested that HAE-4 also reduced fibrillar plaque load, but the effect was not significant (Figure 2, A and C). However, HAE-4 significantly reduced insoluble A $\beta_{40}$ (Figure 2D) and $A \beta_{42}$ (Figure 2E) in the guanidine fraction of the cerebral cortex, as compared with both the PBS- and IgG2abtreated control groups at the same age. There were no signifi- 
A

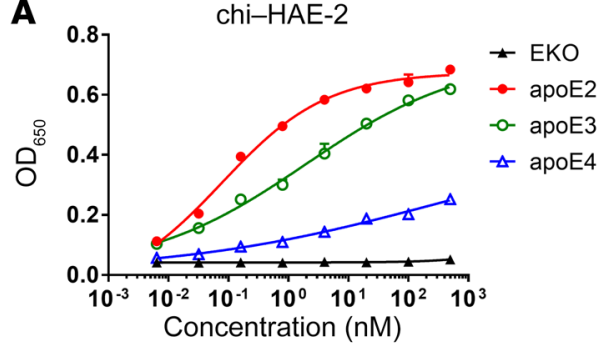

C

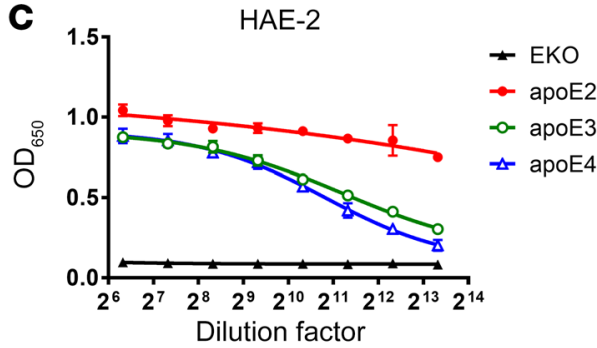

$\mathbf{E}$

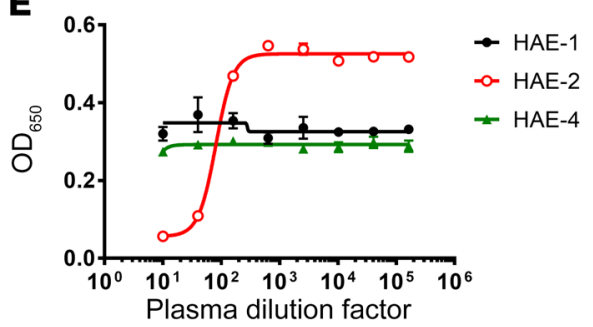

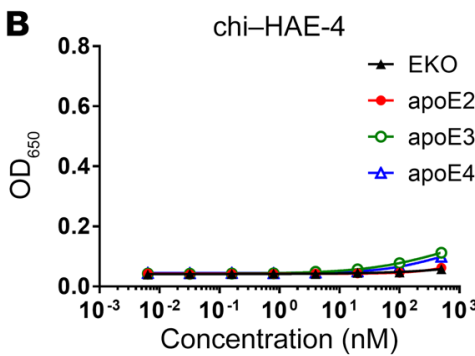
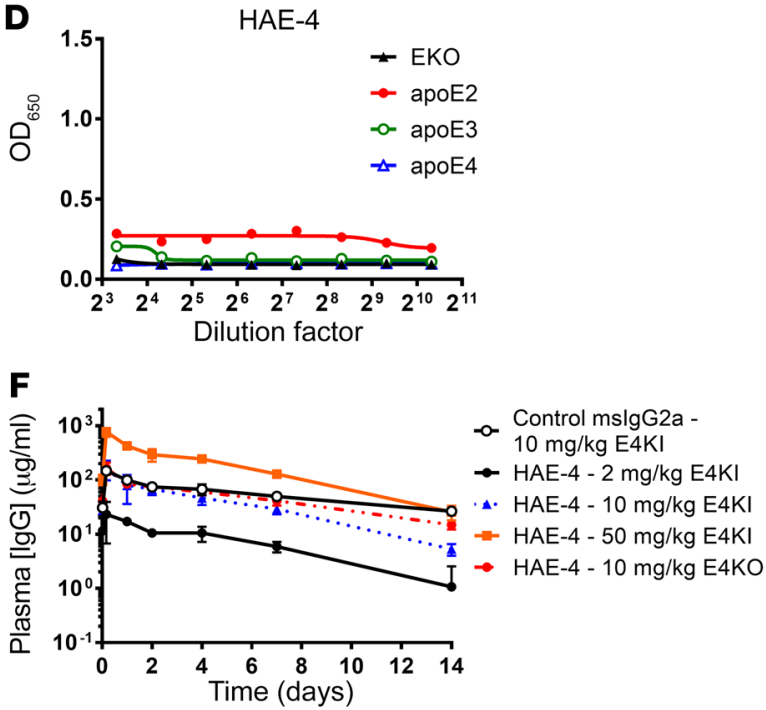

Figure 3. Binding profile of HAE-1, HAE-2, and HAE-4 with lipidated apoE. (A and B) Antibody binding to mouse plasma from EKO, APOE2, APOE3, and APOE4 mice was coated onto the plates. Titrations of chi-HAE-2 and chi-HAE-4 were incubated and antibody that was captured was detected with HRP-goat anti-human IgC antibody. (C and D) HAE-2 or HAE-4 was immobilized on the plate followed by the addition of plasma from mice with different genotypes. apoE, captured from the plasma, was detected with HRP-goat polyclonal anti-apoE. (E) Plasma inhibition of anti-apoE binding to immobilized recombinant apoE4. HAE-1 (50 nM), HAE-2 (4 nM), and HAE-4 (50 nM) were preincubated with serially diluted plasma from APOE4-KI mice and then added to plates coated with recombinant apoE4. The HAE antibodies bound to the plates were detected with HRP-goat anti-mouse IgG antibodies. (F) Plasma antibody concentrations of HAE-4 or control lgG following i.p. injection into APOE4-KI or EKO mice. HAE-4 was dosed at 2 mg/kg, 10 mg/kg, and $50 \mathrm{mg} / \mathrm{kg}$ and plasma samples were collected by submandibular puncture. Control murine IgG2a (mslgG2a) was anti-Her2 and dosed at 10 mg/kg. Quantification of dosed antibodies in plasma was by antigen-capture ELISA using coated recombinant apoE4 to detect HAE-4, with recombinant Her2 used to detect the control IgG.

cant changes in levels of brain apoE, plasma apoE, or plasma $\mathrm{A} \beta$ after HAE-1 or HAE-4 treatment relative to the control groups at the same age (Supplemental Figure 2). A possible reason that the i.p. injection of $50 \mathrm{mg} / \mathrm{kg} \mathrm{HAE}-4$ had a somewhat greater effect on reducing $A \beta$ levels than the results obtained with chronic i.c.v. infusion (Supplemental Figure 1) is that the concentration of HAE- 4 in the brain after 6 weeks of treatment was significantly higher with the i.p. injection paradigm $(6.37 \pm 2.05$ $\mathrm{ng} / 100 \mathrm{mg}$ tissue, $n=13)$ than the i.c.v. infusion paradigm $(2.16$ $\pm 0.7 \mathrm{ng} / 100 \mathrm{mg}$ tissue, $n=9)(P=0.001)$. Unlike HAE-4, HAE1 had no significant effect on lowering $A \beta$ levels. This may be due to the fact that HAE-1 is an IgG1 antibody whereas HAE4 is an IgG2ab antibody. Mouse IgG1 antibodies bind poorly to Fc $\gamma R$ 1 receptors (16). In a separate experiment performed under the same conditions, HAE- 3 did not change the $A \beta$ plaque load, fibrillar plaque load, or insoluble $A \beta$ in the brain compared with PBS-treated mice (Supplemental Figure 3). This is likely due to the very short half-life of HAE-3 in the blood following i.p. injection (see below). In another cohort of APPPS1-21/APOE4 animals, we performed a dose-response study with HAE-4. At 2 months of age, the mice (mixed sexual phenotype, $n=17-18$ / group) received weekly i.p. injections of control IgG2ab (50 mg/ $\mathrm{kg}$ ), anti-A $\beta$ antibody $\mathrm{HJ} 3.4$, which recognizes amyloid plaques (50 mg/kg), or HAE-4 (2, 10, and $50 \mathrm{mg} / \mathrm{kg}$ ) for 2.5 months prior to sacrifice. There was a significant dose-dependent effect of $\mathrm{HAE}-4$ on reducing insoluble $\mathrm{A} \beta_{40}$ and $\mathrm{A} \beta_{42}$ levels in the brain (Supplemental Figure 4).

Binding profile of HAE antibodies to lipidated apoE. Given that the antibodies were chronically injected into the peripheral compartment at high levels, we were somewhat surprised that HAE-1 and HAE-4 did not affect levels of plasma apoE or brain apoE, as apoE is abundant in both locations. This prompted us to ask whether some of the anti-apoE antibodies bound differentially to lipidated apoE versus other forms of the protein. In order to assess binding to lipidated apoE, we characterized the binding of $\mathrm{HAE}$ antibodies to plasma-derived apoE, the majority of which is lipidated. Plasma that contained intact plasma lipoproteins from apoE-KO (EKO), APOE2-, APOE3-, and APOE4-KI mice was 

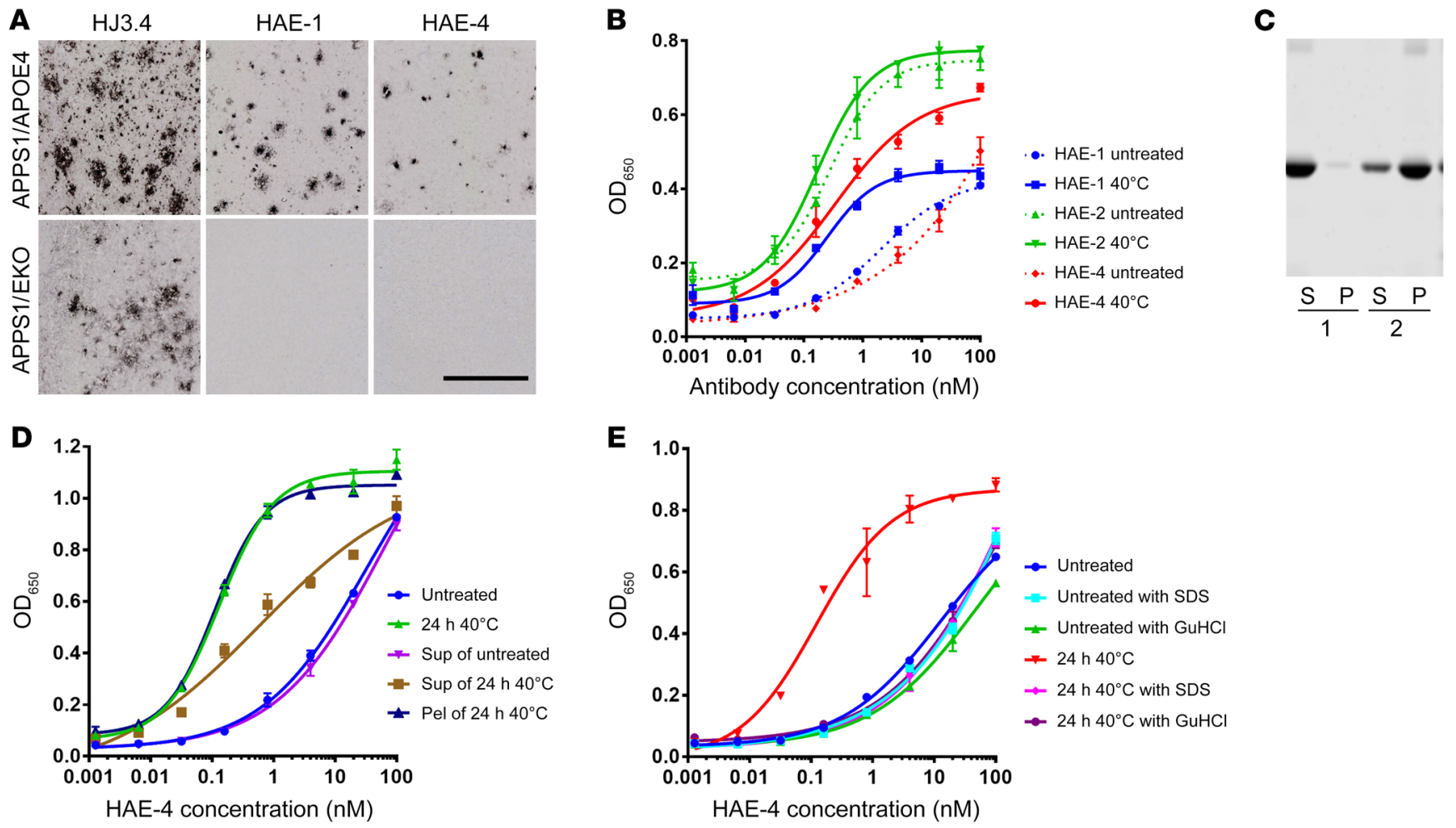

Figure 4. HAE-1 and HAE-4 staining of amyloid plaques in unfixed mouse brain sections and specificity for heat-induced aggregates of apoE4. (A) Unfixed frozen brain sections from APPPS1-21/APOE4 or APPPS1-21/EKO mice were stained with anti-A $\beta$ antibody HJ3.4 and anti-apoE antibodies HAE-1 and HAE-4. Scale bar $=400 \mu \mathrm{m}$. (B) Binding of HAE-1, HAE-2, and HAE-4 to untreated recombinant apoE4 (untreated) or apoE4 that had been incubated at $40^{\circ} \mathrm{C}$ for 24 hours $\left(40^{\circ} \mathrm{C}\right)$. (C) Incubation of apoE4 at $40^{\circ} \mathrm{C}$ for 24 hours results in the formation of aggregates recovered in the pellet fraction following ultracentrifugation at $186,000 \mathrm{~g}$ for 1 hour. Lane 1, untreated apoE4. Lane 2, apoE4 that had been incubated at $40^{\circ} \mathrm{C}$ for 24 hours. Supernatant (S) and pellet (P) from ultracentrifugation were resolved on SDS-PAGE and stained by Coomassie blue. (D) Binding of HAE-4 to different preparations of apoE4 immobilized at the same concentration $(0.5 \mu \mathrm{g} / \mathrm{ml})$ on the ELISA plate. Sup of untreated: supernatant fraction of untreated apoE4 from ultracentrifugation. Sup/Pel of 24 hours $40^{\circ} \mathrm{C}$ : supernatant/pellet fraction of apoE4 incubated at $40^{\circ} \mathrm{C}$ for 24 hours. (E) Binding of HAE-4 to untreated apoE4 and apoE4 that had been incubated at $40^{\circ} \mathrm{C}$ before and after denaturation by $1 \%$ SDS or $4 \mathrm{M}$ guanidine $\mathrm{HCl}$.

coated on ELISA plates. Chimeric anti-apoE antibodies HAE-2 (chi-HAE-2) and HAE-4 (chi-HAE-4), which consist of mouse variable regions fused to human constant regions, were added to the plasma-coated plates, and bound chi-HAE-2 or chi-HAE-4 was detected using anti-human IgG. Chi-HAE-2 bound to plasma containing lipidated apoE2, apoE3, and apoE4 (Figure 3A). In contrast, chi-HAE-4 did not bind strongly to plasma containing lipidated apoE2, apoE3, and apoE4 (Figure 3B). To rule out that the coating of apoE from plasma onto plates alters its conformation and its possible detection by the apoE antibodies, we then performed a sandwich ELISA by coating HAE-2 or HAE-4 onto the plates, incubating with plasma from EKO, APOE2, APOE3, and APOE4 mice, and detecting with a polyclonal anti-apoE antibody. Lipidated apoE2, apoE3, and apoE4 in the respective plasma samples was captured by HAE-2 (Figure 3C) but not HAE-4 (Figure 3D). Next, we tested the ability of plasma to inhibit the binding of HAE-1, HAE-2, and HAE-4 to immobilized recombinant nonlipidated apoE4. Antibodies were preincubated with serially diluted plasma from APOE 4 mice and subsequently added to the recombinant apoE4-coated plate. The results showed that plasma inhibited the binding of HAE-2 to immobilized apoE4, suggesting that HAE-2 bound both lipidated and nonlipidated forms of apoE.
In contrast, plasma did not inhibit the binding of HAE-1 or HAE4 , suggesting that these antibodies do not bind lipidated forms of apoE (Figure 3E).

The difference in ability to recognize plasma-derived apoE was further supported by the pharmacokinetic profiles of HAE-1, HAE-2, HAE-3, and HAE-4. Following i.p. injection $(10 \mathrm{mg} / \mathrm{kg})$ into APOE 4 mice, HAE-2 and HAE-3 were rapidly cleared from plasma within 4 hours (Supplemental Figure 5), whereas HAE-4 was present in the plasma 14 days after injection at levels similar to a control mouse IgG2a antibody (Figure 3F). The likely targetmediated clearance observed with $\mathrm{HAE}-2$ and $\mathrm{HAE}-3$ suggests that these antibodies bind to plasma-derived lipidated apoE, which is abundant in the plasma $(\sim 50 \mu \mathrm{g} / \mathrm{ml})$, whereas HAE- 1 and HAE-4 do not. HAE-1 and HAE-4 appear to be selective for nonlipidated apoE, which is low or absent in plasma.

Binding of HAE-4 to plaque and aggregated apoE. Using unfixed frozen brain sections from APPPS1-21/APOE4 or APPPS1-21/ EKO mice, we evaluated whether HAE-1 and HAE-4 could recognize apoE in the brain (Figure $4 \mathrm{~A}$ ). The presence of $\mathrm{A} \beta$ plaques was confirmed with $A \beta$ immunostaining using anti-A $\beta$ antibody HJ3.4 on sections from the same brain. Given the lower total signal seen by SPR for HAE- 1 and HAE- 4 compared with HAE-2 (Fig- 
A
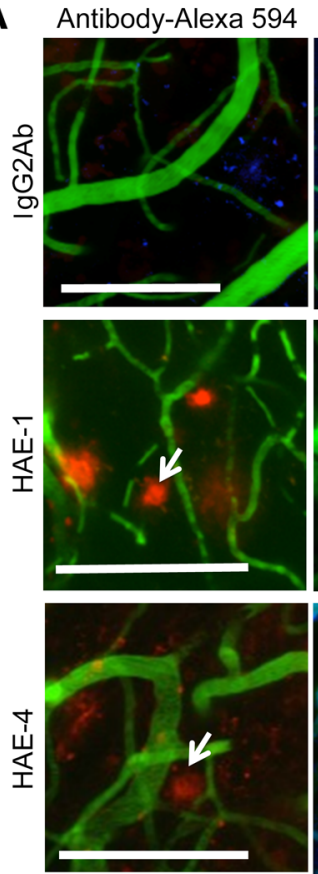

Methoxy
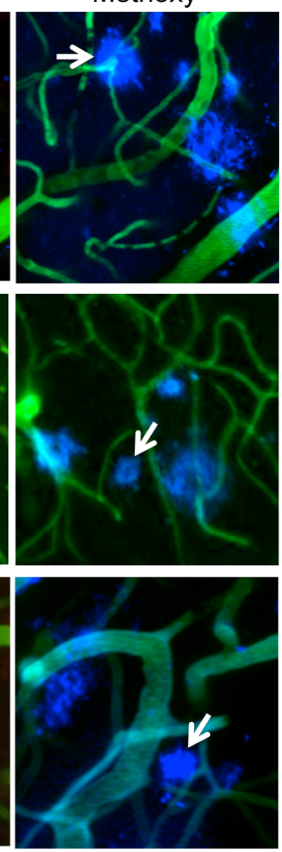

MERGE
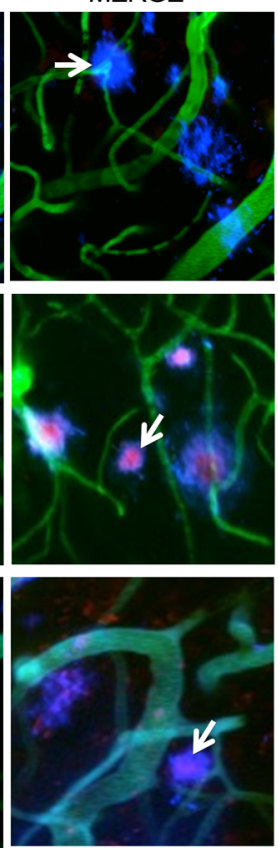

B
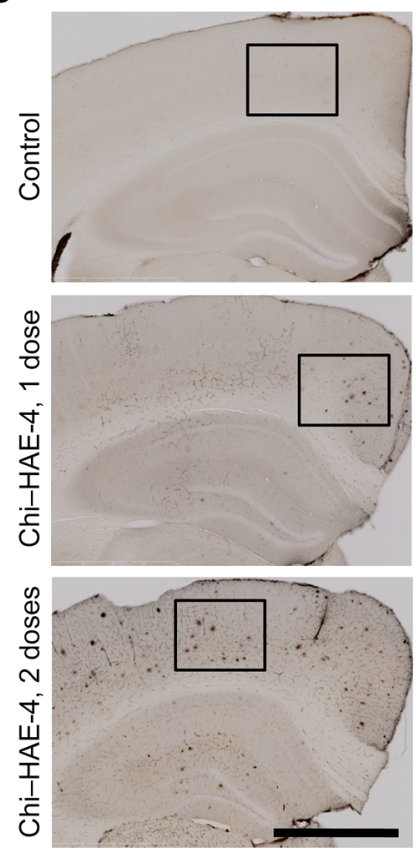
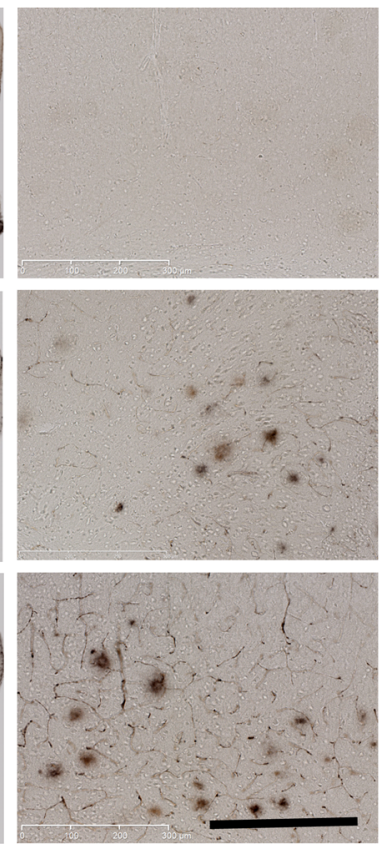

Figure 5. Binding of HAE-1, HAE-4, and control antibody to human apoE4 in the brains of living mice. (A) Control lgG2ab ( $n=7$ ), HAE- 1 ( $n=5$ ), and HAE-4 $(n=6)$ conjugated with Alexa 594 were applied directly onto the surface of the brain in living APPPS1-21/APOE4 mice that were 6 months of age, and antibody localization was observed using 2-photon microscopy. Amyloid was labeled using methoxy-X04. The signal from Alexa 594 and methoxy-X04 was merged (MERGE) to show the colocalization of antibodies and plaques. (B) Control human IgG ( $n=2$ ) or chi-HAE-4 at 50 mg/kg body weight was injected i.p. in 1 dose ( 0 hour, $n=3$ ) or 2 doses ( 0 and 48 hour, $n=3$ ). APPPS1-21/APOE4 mice were sacrificed 48 hours after final injection. The antibodies in the brain were detected by biotinylated rabbit anti-human IgG followed by DAB. Left panel, bar = 1 mm. Right panel, high-power image of the indicated areas shown in the left panel; bar $=300 \mu \mathrm{m}$.

ure $1, \mathrm{E}-\mathrm{G})$, we hypothesized that HAE-1 and HAE-4 bound to a less-abundant subspecies of the recombinant apoE 4 that had been immobilized on the sensor chip. To assess whether HAE- 4 binds to aggregated forms of apoE, we compared the binding of HAE-1, HAE-2, or HAE-4 to untreated apoE4 or apoE4 preincubated overnight at $40^{\circ} \mathrm{C}$. HAE-2 bound to both forms of apoE similarly, whereas the binding of HAE- 1 and $\mathrm{HAE}-4$ to heat-treated apoE was dramatically enhanced (Figure 4B). HAE-1 preferentially binds to nonlipidated apoE 4 and HAE-4 preferentially binds to nonlipidated apoE3 and apoE4. The binding preferences of HAE- 1 to apoE4 and HAE-4 to apoE3 and apoE4 are retained with heat treatment (Supplemental Figure 6). In other words, HAE-1 and HAE-4 show strong preferential binding to aggregated apoE4 (HAE-1) or to apoE3 and apoE4 (HAE-4). Heat treatment of apoE resulted in the formation of apoE aggregates that could be recovered in the pellet fraction following ultracentrifugation at $186,000 \mathrm{~g}$ for 1 hour (Figure 4C). See complete unedited blots in the supplemental material. Compared with apoE recovered from the supernatant fraction, HAE- 4 was able to bind apoE from the pellet fraction much more readily (Figure 4D). This suggests that HAE-4 preferentially recognizes aggregated apoE generated by heat treatment. Solubilization of the apoE aggregates using 1\% SDS or $4 \mathrm{M}$ guanidine eliminated the preference of $\mathrm{HAE}-4$ for heat-induced apoE aggregates (Figure 4E). In summary, these data suggest that HAE4 preferentially binds to aggregated forms of apoE, which may be the predominant form of apoE found in the plaques of APPPS1-21 mouse and human brain sections.
Binding of HAE-4 to brain apoE in vivo. Next, we tested whether $\mathrm{HAE}-1$ and $\mathrm{HAE}-4$ could bind to apoE in the brains of living mice. A mouse IgG2ab (control), HAE-1, and HAE-4 were conjugated with Alexa 594 and applied to the brain surface $(40 \mu \mathrm{lof} 1 \mathrm{mg} / \mathrm{ml})$ of 6-month-old APPPS1-21/APOE4 mice. Antibody localization was monitored through a cranial window using 2-photon microscopy (Figure 5A). HAE-1 and HAE-4 were found to localize to amyloid plaques. To test whether peripherally administered apoE antibodies could enter the brain and bind to apoE in plaques, chi-HAE-4 or control human IgG were i.p. injected $(50 \mathrm{mg} / \mathrm{kg}$ ) for 1 or 2 doses into 5- to 6-month-old APPPS1-21/APOE4 mice. Two days after the final injection, chi-HAE- 4 was detected bound to plaques in the dosed mice (Figure 5B). This indicated that chi-HAE-4 was able to enter the brain and bind to apoE in the $\mathrm{A} \beta$ plaques in living animals.

The effects of HAE-1 and HAE-4 on microglial activation and $A \beta$ deposition: requirement of a microglial response. To determine whether HAE-1 and HAE-4 antibodies can increase activated microglia or infiltrating monocytes, we quantified $\mathrm{CD} 45^{+}$cells relative to the amount of fibrillar plaques after short-term treatment of HAE- 1 and HAE- 4 antibodies (4 doses by i.p. injection every 3 days) in 4-month-old APPPS1-21/APOE4 mice that already had existing plaques (Figure 6A and Supplemental Figure 7). After acute passive immunization, HAE- 1 had no effect on the amount of $\mathrm{CD} 45^{+}$cells, whereas HAE- 4 significantly increased the CD $45^{+}$ cells compared with the controls (Figure 6A). Interestingly, there was no increase in the number of Iba- $1^{+}$cells around plaques following HAE-4 treatment $\left(1.02 \times 10^{-4} \pm 8.63 \times 10^{-6}\right.$ cells $/ \mu \mathrm{m}^{3}$ for 
A
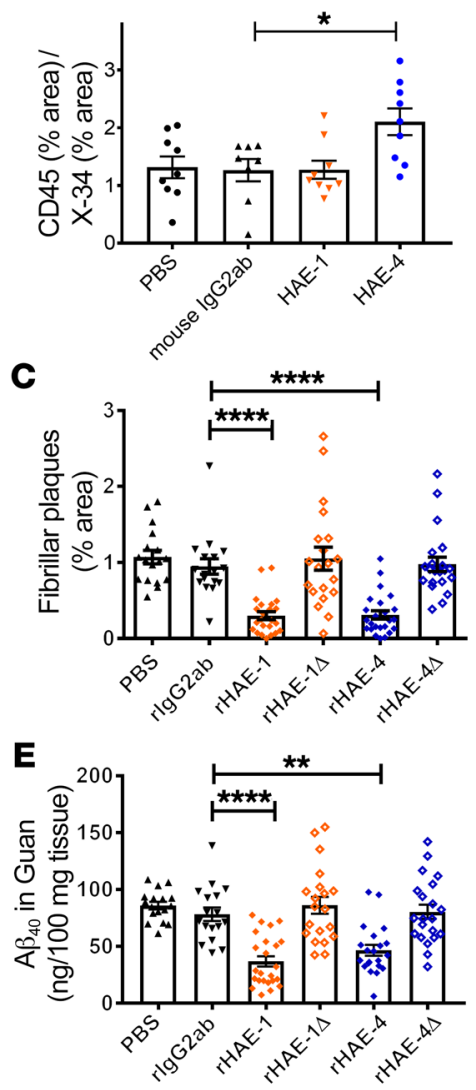
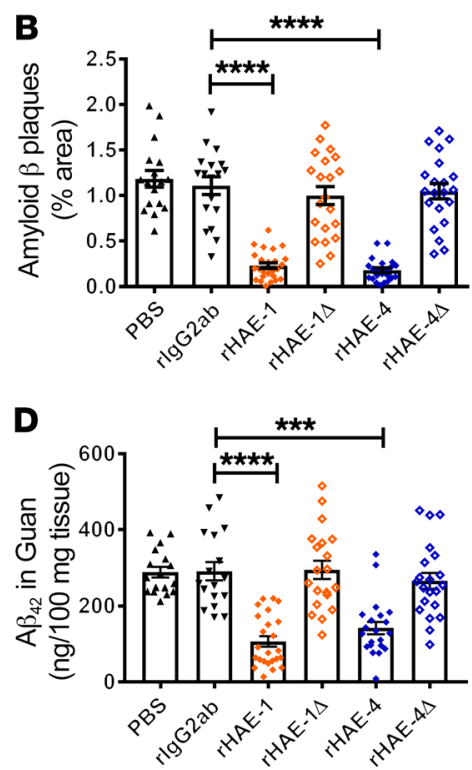

Figure 6. Reduction of plaques by HAE-1 and HAE-4 requires effector function. (A) At the age of 4 months, the APPPS1-21/ APOE4 mice received 4 i.p. injections of $50 \mathrm{mg} / \mathrm{kg}$ of antibodies every 3 days. The mice were sacrificed 24 hours after the final injection and the fibrillar plaques were stained with X-34 and the activated microglia was stained with CD45. The ratio of percentage of area covered by CD45 staining/percentage of area covered by $\mathrm{X}-34$ staining was quantified (equal numbers of male and female mice, $n=8-9 /$ group). (B-E) APPPS1-21/ APOE4 mice were injected at day PO with AAV 2/8 into the lateral ventricle (equal numbers of male and female mice, $n=17-25$ /group). AAV $2 / 8$ is able to express and secrete full-length $\mathrm{HAE}-1$ and $\mathrm{HAE}-4$ antibodies as well as the same constructs with a D265A mutation in the $\mathrm{Fc}$ domain (HAE-1 $\triangle$ and $\mathrm{HAE}-4 \Delta$ ). At the age of 3.5 months, the $A \beta$ plaques (B) were stained with antibody $\mathrm{HJ} 3.4$, the fibrillar plaques were stained with $X-34(\mathbf{C})$, and the insoluble $A \beta_{42}(\mathbf{D})$ and $A \beta_{40}$ (E) were measured by ELISA. One-way ANOVA followed by Tukey's $t$ test was performed to compare different groups shown in A-E. Data are mean \pm SEM. ${ }^{*} P<0.05,{ }^{* *} P<0.01$, ${ }^{* * *} P<0.001,{ }^{* * *} P<0.0001$. control, $n=7$ vs. $1.29 \times 10^{-4} \pm 2.09 \times 10^{-5}$ cells $/ \mu \mathrm{m}^{3}$ for HAE- $4, n=$ 7) suggesting that together with the CD 45 data the total number of either microglia or infiltrating monocytes around plaques was not increased, but the cells present were in a more activated state. Although HAE- 1 and HAE-4 can bind to apoE in plaques, antibody-driven plaque reduction may be dependent upon microglial activation. In order to assess whether microglia are required for an HAE-1- or HAE-4-dependent decrease in $\mathrm{A} \beta$ accumulation, we used AAV serotype 2/8 to drive the expression and secretion of full-length HAE-1 and HAE-4 antibodies with and without a D265A mutation in the Fc domain. The D265A mutation removes virtually all binding to $\mathrm{F} c \gamma$ receptors and prevents these antibodies from effectively activating microglia (17). AAV vectors expressing these antibodies (all with an IgG2ab Fc domain or a control IgG2ab antibody) were injected into APPPS1-21/APOE4 mice at PO. Mice were then sacrificed at 3.5 months of age and assessed. We measured the levels of HAE- 1 and HAE- 4 antibodies with and without the D265 mutation in the cortex and found that they were all expressed at similar levels with no statistical differences among the different antibodies (Supplemental Figure 8). The levels of HAE-1 and HAE-4 were much higher in the cortex with AAV-mediated expression (over $20 \mathrm{ng} / 100 \mathrm{mg}$ tissue) in the mice at 3.5 months of age than they were after 6 weeks of i.p. injections ( $6 \mathrm{ng} / 100 \mathrm{mg}$ tissue). Following AAV-mediated expression of HAE-1 or HAE-4 in APPPS1-21/APOE4 mice, there was a strong and significant reduction of $A \beta$ immunostaining, fibrillar $A \beta$, and insoluble $A \beta_{40}$ and $A \beta_{42}$ as compared with control antibody-treated mice (Figure 6, B-E). In contrast, the AAVs expressing HAE- 1 or
HAE-4 with a D265A mutation had no effect on $A \beta$ immunostaining, fibrillar $A \beta$, and insoluble $A \beta_{40}$ or $A \beta_{42}$ relative to the control antibody (Figure 6, B-E). This strongly suggests that anti-apoE antibodies that bind to apoE in $A \beta$ plaques decrease $A \beta$ accumulation through an Fc $\gamma$ R-mediated mechanism.

\section{Discussion}

In the present study, we report that both i.c.v. and i.p. administration of the anti-human apoE antibody HAE-4 (specific for apoE3 and apoE4) into APP transgenic mice expressing human apoE4 significantly reduced $A \beta$ plaque load and $A \beta$ accumulation in the brain. HAE-4 preferentially binds to nonlipidated forms of apoE as compared with lipidated apoE, which is the major form of apoE in vivo. When monomeric and aggregated forms of apoE were compared, HAE-4 preferentially bound to apoE aggregates. HAE-4 bound to apoE in amyloid plaques after direct application to the brain surface or after peripheral administration into living animals. Importantly, HAE-4 did not significantly alter the levels of total apoE in the brain or plasma, and both HAE- 1 and HAE- 4 were able to reduce plaque accumulation by direct central administration or expression via AAV. This effect appears to require microglial activation, as the corresponding antibodies with mutations in the Fc effector domain of the antibodies that ablate Fc $\gamma$ receptor binding are not effective in plaque clearance. Overall, these findings demonstrate that certain anti-apoE antibodies selective for nonlipidated, aggregated apoE, such as HAE-1 and HAE-4, can preferentially bind to apoE in plaques and have very similar effects to anti-A $\beta$ antibodies that also bind to and decrease $A \beta$ accumula- 
tion via a microglial-mediated clearance mechanism $(18,19)$. Further studies will be required to determine potential advantages and disadvantages to this approach versus the use of anti- $\mathrm{A} \beta$ antibodies as a treatment modality.

Although apoE has been established as the strongest genetic factor for LOAD for more than 20 years, the mechanism(s) by which apoE modifies AD pathogenesis is still not entirely clear. However, a large body of literature shows that apoE affects $\mathrm{AD}$ at least in part through exacerbating $A \beta$ aggregation and reducing its clearance $(7,20,21)$. The effects of apoE isoform, level, or lipidation status on $\mathrm{A} \beta$ pathology have been extensively studied in vitro and in vivo $(7,21)$. Whether increasing or decreasing of apoE function or expression is beneficial for reducing $\mathrm{A} \beta$ pathology has long been debated. Overexpression of apoE2 using viral vectors reduces $A \beta$ pathology, whereas overexpression of apoE4 increases $A \beta$ pathology in APP transgenic mice (22-25). Increasing apoE4 expression prior to but not after $A \beta$ deposition enhances plaque burden (26). Genetic removal of endogenous murine apoE or reduction of apoE with antisense oligonucleotides prior to the onset of $A \beta$ deposition decreases $A \beta$ plaque load and fibrillar plaques in APP transgenic mice (27-29). Lowering apoE levels after $A \beta$ deposition had started had no effect on A $\beta$ levels (29). APP mice that express 1 copy of human apoE have markedly less $A \beta$ pathology as compared with those expressing 2 copies of the same apoE isoform $(30,31)$. Blocking the interaction between apoE and $A \beta$ using $A \beta 12-28 p$ suppresses $A \beta$ plaque deposition in APP transgenic mice (32). Taken together, only the overexpression of the apoE2 isoform appears beneficial, at least with respect to reducing $\mathrm{A} \beta$ accumulation. Based on the effects we describe, the ability to target specific pools of apoE might be an ideal therapeutic strategy to address the more than $60 \%$ of the patients with LOAD who are apoE 4 carriers. Importantly, this approach of specifically targeting apoE in plaques allows for a direct antibody-mediated decrease in $\mathrm{A} \beta$ accumulation, in contrast to the alternate approach of simply lowering apoE levels. Whether anti-apoE antibodies such as HAE4 will clear existing apoE-containing plaques will need to be tested in future studies.

In previous studies, we found that anti-mouse apoE antibody $\mathrm{HJ} 6.3$ reduced $\mathrm{A} \beta$ plaque load in APPPS1 mice expressing murine apoE $(10,11)$. In those studies, we were not able to determine the mechanism whereby anti-apoE modulated plaque pathology. To better explore this issue, as well as to develop antibodies that bind to human apoE, we generated a series of anti-human apoE antibodies and assessed their efficacy and mechanism of action in mice that develop $\mathrm{A} \beta$ deposition and express human apoE4. We focused most of these studies on antibody HAE-4, as it was most effective at reducing plaques when given peripherally. We first analyzed the apoE levels in the treated animals. Similar to the findings in our previous study using anti-mouse apoE antibody HJ6.3 $(10,11)$, peripheral administration of HAE- 4 did not change the level of plasma apoE or the level of total brain apoE in the tissue lysates. Therefore, the effects of $\mathrm{HAE}-4$ on $\mathrm{A} \beta$ plaques were not mediated simply by lowering total apoE levels. It was previously reported that a plaque-specific $\mathrm{A} \beta$ antibody ( $\mathrm{mE} 8$ ) that targets only pyro-glutamated $A \beta$, a small proportion of $A \beta$ in amyloid plaques, was able to localize to $A \beta$ plaques and remove them by triggering microglia-mediated phagocytosis (19). In the present study, we found that HAE-4 is able to bind to apoE in amyloid plaques, both on unfixed brain sections and in the brain of living APPPS1-21/APOE4 mice following central or peripheral injection. Further, we found that the ability of HAE-4 to decrease plaque accumulation was dependent on microglial activation. This suggests that the key features of this antibody's ability to decrease $A \beta$ plaques are its selectivity toward binding a conformation of apoE in amyloid plaques, specifically apoE aggregates, and its ability to direct microglial-driven apoE-amyloid phagocytosis.

To better understand whether the antibody most effective at decreasing plaques was binding to a particular form of apoE that was selectively present in plaques and not normally found in brain or blood, we assessed the binding affinity of HAE-4 to lipidated verus nonlipidated apoE as well as monomeric versus aggregated apoE. We found that HAE-4 preferentially interacts with a nonlipidated form of apoE as compared with lipidated forms that would be present in plasma, cerebrospinal fluid, or the extracellular space of the brain. This is the first report to our knowledge that suggests that targeting a specific conformational form of apoE reduces $A \beta$ pathology in APP transgenic mice. Given that nonlipidated apoE is only a small percentage of total apoE in vivo (33), targeting this pool of apoE may underlie the lack of effect on apoE levels or lipid metabolism. Reduced binding to lipidated apoE contributes to the much longer plasma half-life of HAE-4 as compared with HAE-2 and HAE-3, which strongly bind lipidated apoE (Figure 3F, Supplemental Figure 5). A longer half-life greatly increases both peripheral and brain exposure of the antibody and enables increased plaque decoration.

Previous work has shown that decreasing $A \beta$ pathology by enhancing apoE lipidation via ATP-binding cassette transporter A1 (ABCA1) has beneficial effects (34-36), and that decreasing apoE lipidation increases $A \beta$ pathology (37). It is possible that poorly lipidated apoE adopts a conformation more prone to aggregation and thus promotes $A \beta$ aggregation. In the present study, we demonstrated that a potent, plaque-reducing antibody may recognize this form of aggregated apoE, which resides in $\mathrm{A} \beta$ plaques in APPPS1-21/APOE4 mice. In the future, the frequency and abundance of HAE-4 immunoreactivity apoE in the brain of patients with $\mathrm{AD}$ will need to be determined.

We found that the effects of 2 different anti-apoE antibodies appear to require microglial-mediated $\mathrm{A} \beta$ phagocytosis, because versions of HAE- 1 and HAE-4 lacking a functional Fc domain of the antibody were not able to decrease $A \beta$ deposition. In addition, in APPPS1-21/APOE4 mice that already had plaques, HAE-4 acute administration substantially increased $\mathrm{CD} 45^{+}$cells around plaques. A variety of anti-A $\beta$ antibodies have been studied for their ability to decrease $A \beta$ plaques both in animal models and in humans. The antibodies shown to have the greatest effects on $\mathrm{A} \beta$ deposition appear to work by binding certain $\mathrm{A} \beta$ species in plaques and then facilitating clearance via microglial phagocytosis $(18,19)$. The mechanism we demonstrate here for HAE-1 and HAE- 4 appears similar, except that these antibodies bind to an $A \beta$-binding molecule (i.e., apoE) and not directly to $A \beta$ itself. The effects we see with these antibodies appear similar to that seen with $\mathrm{A} \beta$ antibodies that lower plaques. Whether the $\mathrm{CD} 45^{+}$ reactive cells are microglia or infiltrating monocytes and to what extent FcR expressed by other cell types in the brain contribute 
to the process of decreasing $A \beta$ accumulation needs to be sorted out in future studies. In addition to efficacy, it will be important to determine whether anti-apoE antibodies with features such as HAE-4 have any potential side effects. We were not able to determine whether there was an increase in microhemorrhages in the APPPS1-21/APOE4 mice following treatment with HAE-4, as there was very little amyloid angiopathy in this mouse model. Future studies addressing this issue may support the development of anti-apoE antibodies for the treatment of AD.

\section{Methods}

Animals. APPPS1-21 mice on a C57BL/6J background (gift from Mathias Jucker, Hertie Institute for Clinical Brain Research, Tubingen, Germany) coexpress human APP with a Swedish mutation (KM670/671NL) and mutant PS1 with the L1669 mutation under the control of a Thy1 promoter (13). APOE2-, APOE3-, and APOE4-knockin mice express APOE E2, E3, and E4 under control of the endogenous mouse regulatory elements on a C57BL/6J background (38). EKO mice were purchased from Taconic. APPPS1-21/APOE4 mice were generated by breeding APPPS1-21 with APOE4 mice. The A $\beta$ plaque pathology in APPPS1-21/APOE4 mice begins at the age of 2 months.

Generation of antibodies. The HAE antibodies were generated by injecting recombinant apoE4 with complete Freund's adjuvant into mice. For an initial screening of antibodies, supernatants from hybridoma cells were added to 96-well plates coated with recombinant apoE4 and the HAE that bound to apoE4 was detected using anti-mouse IgG HRP. The antibodies that performed well in the initial screening were further characterized and selected for in vivo studies. For the in vivo study, antibodies were generated from cultured hybridoma cells and purified on a protein G column. All HAE antibodies utilized in vivo contained similar amounts of endotoxin as compared with control antibodies. All 4 anti-apoE antibodies studied are mouse IgGs. HAE-1 is an IgG1. HAE-2, HAE-3, and HAE-4 are IgG2ab subtypes.

apoE binding ELISAs. Recombinant apoE (Leinco Technologies) was coated to half-area 96-well plates at $0.5 \mu \mathrm{g} / \mathrm{ml}$ in PBS overnight at $4^{\circ} \mathrm{C}(25 \mu \mathrm{l} /$ well $)$. After 3 washes with PBS, the wells were blocked with $1 \%$ BSA-PBS for 1 hour at room temperature with shaking at $500 \mathrm{rpm}$. The blocked wells were washed once with PBS and subsequently loaded with HAE antibodies at serial concentrations (starting at $300 \mathrm{nM}$ with 3-fold dilutions thereafter). Bound HAE antibodies were detected with HRP-labeled goat anti-mouse IgG (catalog 115-035-003, Jackson ImmunoResearch Laboratories) and visualized with tetramethylbenzidine (TMB) substrate at $\mathrm{OD}_{450}$ (reaction stopped with $4 \mathrm{~N} \mathrm{H}_{2} \mathrm{SO}_{4}$ ) or $\mathrm{OD}_{650}$ (reaction stopped with BioFX stop solution, Surmodics). For experiments where apoE aggregation was first induced by heating, recombinant apoE4 at a concentration of $1 \mathrm{mg} / \mathrm{ml}$ was first heated at $40^{\circ} \mathrm{C}$ for 24 hours and then coated directly to ELISA plates at 0.5 $\mu \mathrm{g} / \mathrm{ml}$. To normalize the amount of apoE coated to the ELISA plates from the supernatant and pellet fractions after heating and ultracentrifugation, the relative amounts of apoE were first assessed by SDS-PAGE followed by a Coomassie blue stain. Heat-induced apoE4 aggregates were denatured in $1 \%$ SDS or $4 \mathrm{M}$ guanidine- $\mathrm{HCl}$ at $95^{\circ} \mathrm{C}$ for 10 minutes and then diluted to $0.5 \mu \mathrm{g} / \mathrm{ml}$ in PBS for coating onto ELISA plates. After overnight incubation at $4^{\circ} \mathrm{C}$, the wells were washed 3 times with PBS to remove any residual SDS or guanidine-HCl. ELISA experiments with heated apoE used HAE antibodies starting at 100 $\mathrm{nM}$ with 5-fold dilutions thereafter.
Surface plasmon resonance. Anti-biotin antibody (catalog 28920233, GE Healthcare) was immobilized on the surface of a CM5 chip (GE Healthcare) through amine coupling. The surface was activated by injection of a mixture of EDC/NHS (1-ethyl-3-[3-dimethylaminopropyl] carbodiimide hydrochloride/ $N$-hydroxysuccinimide) (GE Healthcare). Anti-biotin antibody was diluted in sodium acetate (pH 5.0 at $12.5 \mu \mathrm{g} /$ $\mathrm{ml}$ ) and injected for 10 minutes at a flow rate of $5 \mu \mathrm{l} / \mathrm{min}$, followed by injection of ethanolamine (GE Healthcare). Recombinant apoE4 was biotinylated using the EZ-Link Sulfo-NHS-LC-Biotin kit (ThermoFisher Scientific). Biotinylated apoE4 was captured on the chip to reach 550 RU (HAE-1 and HAE-4) or 280 RU (HAE-2). A range of serially diluted antibodies (1.2 nM-100 nM for HAE-2; $12 \mathrm{nM}-1,000 \mathrm{nM}$ for HAE-1 and HAE-4) was injected at a flow rate of $30 \mu \mathrm{l} / \mathrm{min}$, and sensorgrams were fitted using the 1:1 Langmuir model.

Intracerebroventricular antibody administration and sample collection. At the age of 2 months, APPPS1-21/APOE4 mice underwent surgical implantation of a subcutaneous osmotic minipump (Alzet, model 2006) connected to a catheter into the left lateral cerebral ventricle (Bregma-0.4 mm, $1.0 \mathrm{~mm}$ lateral to midline, $2.5 \mathrm{~mm}$ below the skull). The apoE antibodies or control antibodies $(2 \mathrm{mg} / \mathrm{ml})$ were filled into the osmotic minipump and continuously infused (i.c.v.) at the speed of $0.15 \mu \mathrm{l} / \mathrm{h}$ for 6 weeks. At the age of 3.5 months, the mice were perfused with ice-cold PBS containing $0.3 \%$ heparin. The right hemibrain was dissected and flash-frozen on dry ice for biochemistry assays. The left hemibrain was fixed in $4 \%$ paraformaldehyde for histological analysis. Serial coronal sections at $50-\mu \mathrm{m}$ thickness were collected from the rostral to the caudal end of each brain hemisphere using a freezing sliding microtome (Leica Biosystems).

Intraperitoneal antibody administration and sample collection. Twomonth-old female APPPS1-21/APOE4 mice were injected i.p. with a weekly dose of $50 \mathrm{mg} / \mathrm{kg}$ body weight of control or HAE anti-apoE antibodies for 7 doses. At the age of 3.5 months, the mice were perfused with ice-cold PBS containing $0.3 \%$ heparin. The right hemibrain was dissected and flash-frozen on dry ice for biochemical assays for $\mathrm{A} \beta$ and apoE. The left hemibrain was fixed in $4 \%$ paraformaldehyde for 48 hours for histological analysis of amyloid plaque load. The fixed brains were sectioned by Multibrain Technology (NeuroScience Associates NSA Labs) at a $50-\mu \mathrm{m}$ thickness. For the dose-response study of HAE-4, another cohort of APPPS1-21/APOE 4 mice received weekly i.p. injections of HAE-4 (2 mg/kg, $5 \mathrm{mg} / \mathrm{kg}$, and $10 \mathrm{mg} / \mathrm{kg})$, anti-A $\beta$ $\mathrm{HJ} 3.4$ (50 mg/kg), or IgG2ab (50 mg/ kg). The cortices were extracted sequentially in PBS and $5 \mathrm{M}$ guanidine and the $A \beta_{40}$ and $A \beta_{42}$ in the guanidine fraction was assessed using ELISA.

ELISA for tissue lysates. To extract $\mathrm{A} \beta$ in different fractions, brain cortices were sequentially homogenized with cold PBS, $1 \%$ Triton-X 100 , and $5 \mathrm{M}$ guanidine buffer in the presence of $1 \times$ protease inhibitor mixture (Roche). The levels of $A \beta_{40}, A \beta_{42}$, and apoE were measured by sandwich ELISA. For $A \beta_{40}$ or $A \beta_{42}$, anti-A $\beta_{35-40}$ HJ2 (produced inhouse) or anti-A $\beta_{37-42} \mathrm{HJ7.4}$ (produced in-house) were used as capture antibodies, and anti-A $\beta_{13-18}$ HJ5.1-biotin (produced in-house) was used as a detecting antibody (39). For apoE ELISA, HJ6.2 (produced in-house) (10) was used as the capture antibody and HJ6.1-biotin (produced in-house) (10) was used as the detecting antibody. Recombinant apoE4 was used as the standard for the apoE ELISA. For assessment of the concentration of rHAE- $1, \mathrm{rHAE}-1 \Delta$, rHAE- 4 , and rHAE- $4 \Delta$ following expression via AAV2/8, recombinant apoE4 was coated on 96-well ELISA plates overnight at $4^{\circ} \mathrm{C}$. After washing, individual puri- 
fied hybridoma-derived and purified HAE- 1 or HAE-4 were added to wells at different concentrations for a standard curve or the PBS-soluble fraction of mouse cortex was added to the wells. Following washing, the antibodies were detected with HRP-coupled anti-mouse IgG.

Immunohistochemistry for $A \beta$ pathology. $\mathrm{A} \beta$ plaques were immunostained using biotinylated anti-A $\beta_{1-13}$ monoclonal antibody $\mathrm{HJ} 3.4 \mathrm{~B}$ (produced in-house) (11). Fibrillar plaques were stained with $0.025 \%$ Thioflavin S (Sigma-Aldrich) or $10 \mathrm{nM} \mathrm{X-34.} \mathrm{Quantitative} \mathrm{analysis} \mathrm{of}$ immunopositive staining was performed as previously described (39). All quantitation of $A \beta$ and Thioflavin S staining was done by an investigator who was blind to both mouse genotype and treatment condition. Briefly, images of immunostained sections were exported with an NDP viewer (Hamamatsu Photonics), converted to 8-bit grayscale using ACDSee Pro 2 software (ACD Systems), thresholded to highlight positive staining, and analyzed using ImageJ software (NIH). Three sections per mouse (Bregma, $-1.4 \mathrm{~mm}$ caudal to Bregma, and $-2.0 \mathrm{~mm}$ caudal to Bregma) were quantified (cortex dorsal to hippocampus) and the average was used to represent each mouse.

Binding of HAE antibodies to lipidated apoE. Blood was collected from the right atrium of 6-week-old, apoE-targeted replacement mice or EKO mice and centrifuged in plasma collection tubes (containing EDTA) at $14,000 \mathrm{~g}$ for 5 minutes. For the plasma-coat ELISA, plasma was diluted 50-fold in PBS and coated to the bottom of half-well ELISA plates overnight at $4^{\circ} \mathrm{C}$. Plates were washed with PBS and blocked in 5\% BSA-PBS for 2 hours. Antibodies were serially diluted 5 -fold (starting at $500 \mathrm{nM}$ ) in 5\% BSA-PBS and incubated for 1 hour at room temperature with shaking (500 rpm). Plates were next washed extensively in PBS and bound antibody was detected with an HRP-goat antihuman secondary antibody (catalog 109-035-003, Jackson ImmunoResearch Laboratories). TMB was used as the chromogenic reagent and reactions were stopped with BioFX stop solution. Plates were read at $\mathrm{OD}_{650}$. Replicate assays were performed and data from 1 experiment in duplicate are shown. For the plasma-capturing experiment, antibodies were coated at $5 \mu \mathrm{g} / \mathrm{ml}$ in half-well ELISA plates overnight at $4^{\circ} \mathrm{C}$. Plates were washed with PBS and blocked in $4 \%$ milk-PBS for 2 hours. Mouse plasma was serially diluted 2-fold (starting at 10x) in $5 \%$ BSA-PBS and incubated on the blocked plates for 3 hours at room temperature and shaken at $500 \mathrm{rpm}$. Plates were next washed extensively in PBS and the captured apoE was detected with polyclonal goat anti-apoE (catalog 178479, Millipore). Anti-goat HRP (catalog 705-035-147, Jackson ImmunoResearch Laboratories) and TMB were used as the detection secondary antibody and chromogenic reagent, respectively. Reactions were stopped with BioFX stop solution and plates were read at $\mathrm{OD}_{650}$. Replicate assays were performed and data from 1 experiment in duplicate are shown. For the plasma competition experiment, recombinant apoE 4 was coated onto half-well plates at $0.5 \mu \mathrm{g} / \mathrm{ml}$ overnight at $4^{\circ} \mathrm{C}$. Plates were washed extensively with PBS and blocked for 1 hour in $4 \%$ BSA-PBS. Antibodies were diluted in $4 \%$ BSA-PBS at double concentrations of the final stock solution and equal volume was added for preincubation with serially diluted plasma (also at a 2-fold final concentration) for 1 hour and shaken at $500 \mathrm{rpm}$. Blocked plates were washed twice and the preincubated antibody/plasma solutions were added to the blocked plates for 1 hour at room temperature with shaking (500 rpm). Plates were next washed extensively in PBS and the bound primary antibody was detected with an HRP-goat anti-mouse secondary antibody (catalog 115-035-003, Jackson ImmunoResearch Laboratories). TMB was used as the chro- mogenic reagent and reactions were stopped with BioFX stop solution. Plates were read at $\mathrm{OD}_{650}$. Replicate assays were performed and data from 1 experiment in duplicate are shown.

Pharmacokinetics of HAE antibodies in mice. Murine HAE-4 and control mouse Ig2a anti-HER2 were injected i.p. at $2 \mathrm{mg} / \mathrm{kg}, 10 \mathrm{mg} /$ $\mathrm{kg}$, and $50 \mathrm{mg} / \mathrm{kg}$, and plasma samples were collected by submandibular puncture at various time points. Assessment of plasma antibody concentrations was performed using coated recombinant apoE4 (5 $\mu \mathrm{g} / \mathrm{ml}$ ) to capture dosed HAE-4, and recombinant Her2 protein (R\&D) $(1 \mu \mathrm{g} / \mathrm{ml})$ to capture the control antibody. Plates were blocked with $3 \%$ BSA in TBS/0.1\% Tween for 1 hour and washed 3 times before incubation with plasma samples at a 1:2500 dilution. Bound antibodies were detected with HRP-anti-mouse IgG (catalog 115-035-003, Jackson ImmunoResearch Laboratories). The standard curve range was 0.49$1000 \mathrm{ng} / \mathrm{ml}$ and fit with a 4-parameter logistic function.

Binding of HAE-1 and HAE-4 to apoE in the unfixed brain tissue. Brain tissue from APPPS1/APOE4 and APPPS1/EKO mice was sectioned at $20-\mu \mathrm{m}$ thickness using a cryotome (ULTRApro 5000, Vibratome) and mounted on slides. The sections were stained with biotinylated HJ3.4, HAE-1, or HAE-4. The antibodies bound to the sections were detected using an $\mathrm{ABC}$ kit (Vector Laboratories) followed by DAB (Sigma-Aldrich).

Cranial window implantation, topical application of antibodies and multiphoton imaging. APPPS1-21/APOE4 mice (5.5-7 months old) were anesthetized under $1.5 \%$ isoflurane, and a $4-\mathrm{mm}$ cranial window was drilled in order to expose the cortex, as previously described (24). After removing the dura matter, $40 \mu \mathrm{l}$ of each antibody stock $(1.0 \mathrm{mg} /$ $\mathrm{ml}$ ), previously conjugated with Alexa 594 (Alexa Fluor 568 Antibody Labeling Kit, ThermoFisher Scientific), was topically applied for 30 minutes. After 2 washes with sterile PBS, a glass coverslip was cemented on the skull in order to seal the window before imaging. Fluorescein dextran (70,000 Da; $12.5 \mathrm{mg} / \mathrm{ml}$ in sterile PBS; Invitrogen) was also injected retroorbitally to provide a fluorescent angiogram. After a first set of images was taken in order to detect the signal from each antibody alone, methoxy- $\mathrm{XO}_{4}(5 \mathrm{mg} / \mathrm{kg})$ was injected intravenously in order to label amyloid plaques as well as cerebral amyloid angiopathy, as previously described (40). In vivo multiphoton imaging was performed using an Olympus FluoView FV1000MPE multiphoton laserscanning system mounted on an Olympus BX61WI microscope and an Olympus $\times 25$ objective (numerical aperture $=1.05$ ). A DeepSee Mai Tai Ti: sapphire mode-locked laser (Mai Tai; Spectra-Physics) generated 2-photon excitation at $800 \mathrm{~nm}$, and detectors containing 3 photomultiplier tubes (Hamamatsu) collected emitted light in the range of 420-460 nm, 495-540 nm, and 575-630 nm. Mice were placed on the microscope stage, heated using a heating pad, and feedback regulation was obtained from a rectal temperature probe (Harvard Apparatus). Z-series images ( $2 \mu \mathrm{m}$ steps, depth of $\sim 200 \mu \mathrm{m}, 512 \times 512$ pixels) were taken to cover a large surface of the window. The laser power was measured and adjusted before each imaging and the settings of the photomultiplier tubes were unchanged throughout the different imaging sessions and between all antibodies applied.

Binding of peripherally administered chi-HAE-4 to apoE in the brain. Chi-HAE-4 and control human IgG were injected i.p. at $50 \mathrm{mg} / \mathrm{kg}$ into 4- to 6-month-old APPPS1-21/APOE4 mice for 1 (0 hour) or 2 (0 and 48 hours) doses and the mice were sacrificed 48 hours after the final injection. The antibodies were detected using biotinylated rabbit antihuman IgG (catalog ab97158, Abcam) followed by DAB. 
Acute injection of HAE antibodies and microglial activation staining. APPPS1-21/APOE 4 mice received 4 doses of antibodies every 3 days at the age of 4 months. Each dose was $50 \mathrm{mg} / \mathrm{kg}$ (administered i.p.). The mice were sacrificed 24 hours after final injection. The fibrillar plaques were stained using X-34. Activated microglia were immunostained using rat-anti CD45 (catalog MCA1388, BioRad) followed by biotinylated goat anti-rat IgG secondary antibody (catalog A10517, Life Technologies). Iba- $1^{+}$cells were detected using rabbit anti-Iba-1 (catalog 019-19741, Wako) followed by donkey anti-rabbit IgG Alexa Fluor 647 (catalog A-31573, ThermoFisher Scientific). For quantification, $3 Z$-stack images were captured per animal on the Nikon $\mathrm{A}^{+} \mathrm{R}^{+}$ confocal microscope using $\times 20$ objective at $1024 \times 1024$ pixel resolution with a $Z$-step size of $1.1 \mu \mathrm{m}$ at $32-\mu \mathrm{m}$ thickness. These images were then processed using Imaris (Bitplane). The coordinates of $\mathrm{X}-34^{+}$plaques and $\mathrm{Iba}-1^{+}$microglia were determined using the Spots function and imported into Matlab (Mathworks). An automated script was used to determine the microglial density around plaque surfaces, and the number of microglia within a $30 \mu \mathrm{m}$ radius was calculated.

Preparation and injection of $A A V 2 / 8$ vectors expressing control antibody and HAE antibodies. The variable regions of heavy and light chain cDNA sequences of control IgG, HAE-1, and HAE-4 were cloned from hybridoma cells. The single open reading frame (ORF) of the heavy chain, Furin cleavage site, P2A, and the light chain was assembled by polymerase chain reaction. All antibody subtypes were switched to mouse IgG2ab. The assembled single ORF was inserted into AAVexpressing vector (serotype 2 ) with a chicken $\beta$ actin promoter. The D265A mutation on the $\mathrm{CH} 2$ region was generated by QuikChange (Agilent Technologies) site-directed mutagenesis. AAV vectors were prepared at Hope Center Viral Vector Core with serotype 8 helper vectors. On PO, APPPS1-21/APOE4 mice (mixed sexual phenotype, $n=17-25 /$ group) received a bilateral i.c.v. injection of $2 \mu \mathrm{l} \mathrm{AAV} \mathrm{vec-}$ tor $\left(1.0 \times 10^{13} \mathrm{vg} / \mathrm{ml}\right)$. The mice were sacrificed at 3.5 months of age. After perfusion with $\mathrm{PBS}$, one brain hemisphere was immersion fixed in $4 \%$ paraformaldehyde for further histological analysis and the other hemisphere was dissected for further biochemical analysis. For histology, the brains were sectioned at $50 \mu \mathrm{m}$ on a freezing sliding microtome. The $\mathrm{A} \beta$ plaques were stained with anti-A $\beta \mathrm{HJ} 3.4$ and the fibrillar plaques were stained using X-34. The cortices were sequentially extracted using PBS and $5 \mathrm{M}$ guanidine, and $\mathrm{A} \beta_{40}$ and $\mathrm{A} \beta_{42}$ were measured by ELISA. The concentration of antibodies in the PBS fraction was also measured by ELISA.

Statistics. Two-tailed Student's $t$ test was used to determine whether there were significant differences between 2 groups unless otherwise specified. One-way ANOVA was used to compare differences among 3 or more groups, followed by Tukey's $t$ test unless otherwise specified. Data in all the figures are mean \pm SEM unless otherwise specified. $P<0.05$ was considered significant.

Study approval. All animal experimental protocols were approved by the Animal Studies Committee at Washington University.

\section{Author contributions}

FL, AL, YZ, APS, JLG, CEGL, GG, NBL, RJW, JDU, PMS, EH, KH, ZKS, MSD, BTH, and DMH designed the research studies. HJ, FL, AL, YZ, APS, NBL, JG, MSD, RJW, and DMH generated and characterized the anti-apoE antibodies. FL, AL, MX, HJ, MBF, RH, KBL, JK, GOR, YZ, JRS, APS, JLG, EPL, and EH conducted experiments and acquired data. FL, AL, MX, YZ, APS, JLG, NBL, RJW, EPL, EH, JG, $\mathrm{KH}, \mathrm{MSD}, \mathrm{ZKS}$, BTH, and DMH analyzed the data. FL, AL, YZ, APS, JLG, NBL, MSD, EH, BTH, RJW, and DMH wrote the manuscript.

\section{Acknowledgments}

This work was supported by a research grant from Denali (to DMH), the NIH (AG R01 AG047644 to DMH and BTH, and grants P50 AG05681, P01 AG03991, and P01 AG026276), and the BrightFocus Foundation (A2013037F to FL). The authors acknowledge Maya Leabman (independent scientific consultant) for discussions on pharmacokinetics, Katherine Jenkins (Denali) for animal support, and Wilbur Song and Marco Colonna (Washington University) for the methods of Iba-1 immunostaining quantification.

Address correspondence to: David M. Holtzman, Department of Neurology, Hope Center for Neurological Disorders, Charles F. and Joanne Knight Alzheimer's Disease Research Center, Washington University School of Medicine, 660 S. Euclid Avenue, Box 8111, St. Louis, Missouri 63110, USA. Phone: 314.747.0644; Email: holtzman@neuro.wustl.edu.
1. Selkoe DJ, Hardy J. The amyloid hypothesis of Alzheimer's disease at 25 years. EMBO Mol Med. 2016;8(6):595-608.

2. Musiek ES, Holtzman DM. Three dimensions of the amyloid hypothesis: time, space and 'wingmen'. Nat Neurosci. 2015;18(6):800-806.

3. Hardy J, Selkoe DJ. The amyloid hypothesis of Alzheimer's disease: progress and problems on the road to therapeutics. Science. 2002;297(5580):353-356.

4. Jonsson T, et al. A mutation in APP protects against Alzheimer's disease and age-related cognitive decline. Nature. 2012;488(7409):96-99.

5. Corder EH, et al. Protective effect of apolipoprotein E type 2 allele for late onset Alzheimer disease. Nat Genet. 1994;7(2):180-184.

6. Strittmatter WJ, et al. Apolipoprotein E: highavidity binding to beta-amyloid and increased frequency of type 4 allele in late-onset familial Alzheimer disease. Proc Natl Acad Sci US A.
1993;90(5):1977-1981.

7. Huynh TV, Davis AA, Ulrich JD, Holtzman DM. Apolipoprotein E and Alzheimer's disease: the influence of apolipoprotein $\mathrm{E}$ on amyloid- $\beta$ and other amyloidogenic proteins. JLipid Res. 2017;58(5):824-836.

8. Namba Y, Tomonaga M, Kawasaki H, Otomo E, Ikeda K. Apolipoprotein E immunoreactivity in cerebral amyloid deposits and neurofibrillary tangles in Alzheimer's disease and kuru plaque amyloid in Creutzfeldt-Jakob disease. Brain Res. 1991;541(1):163-166.

9. Wisniewski T, Frangione B. Apolipoprotein E: a pathological chaperone protein in patients with cerebral and systemic amyloid. Neurosci Lett. 1992;135(2):235-238.

10. Kim J, et al. Anti-apoE immunotherapy inhibits amyloid accumulation in a transgenic mouse model of A $\beta$ amyloidosis. J Exp Med. 2012;209(12):2149-2156.
11. Liao F, et al. Anti-ApoE antibody given after plaque onset decreases $A \beta$ accumulation and improves brain function in a mouse model of $A \beta$ amyloidosis. J Neurosci. 2014;34(21):7281-7292.

12. Sullivan PM, et al. Targeted replacement of the mouse apolipoprotein $\mathrm{E}$ gene with the common human APOE3 allele enhances diet-induced hypercholesterolemia and atherosclerosis. J Biol Chem. 1997;272(29):17972-17980.

13. Radde R, et al. Abeta42-driven cerebral amyloidosis in transgenic mice reveals early and robust pathology. EMBO Rep. 2006;7(9):940-946.

14. Liao F, et al. Murine versus human apolipoprotein E4: differential facilitation of and co-localization in cerebral amyloid angiopathy and amyloid plaques in APP transgenic mouse models. Acta Neuropathol Commun. 2015;3:70.

15. Koenigsknecht-Talboo J, et al. Rapid microglial response around amyloid pathology after systemic anti-A $\beta$ antibody administration in PDAPP 
mice. J Neurosci. 2008;28(52):14156-14164.

16. Guilliams M, Bruhns P, Saeys Y, Hammad H, Lambrecht BN. The function of Fc $\gamma$ receptors in dendritic cells and macrophages. Nat Rev Immunol. 2014;14(2):94-108.

17. Baudino L, et al. Crucial role of aspartic acid at position 265 in the $\mathrm{CH} 2$ domain for murine $\operatorname{IgG} 2 \mathrm{a}$ and $\operatorname{IgG} 2 \mathrm{~b}$ Fc-associated effector functions. JImmunol. 2008;181(9):6664-6669.

18. Sevigny J, et al. The antibody aducanumab reduces A $\beta$ plaques in Alzheimer's disease. Nature. 2016;537(7618):50-56

19. Demattos RB, et al. A plaque-specific antibody clears existing $\beta$-amyloid plaques in Alzheimer's disease mice. Neuron. 2012;76(5):908-920.

20. Castellano JM, et al. Human apoE isoforms differentially regulate brain amyloid- $\beta$ peptide clearance. Sci Transl Med. 2011;3(89):89ra57.

21. Kim J, Basak JM, Holtzman DM. The role of apolipoprotein E in Alzheimer's disease. Neuron. 2009;63(3):287-303.

22. Hu J, Liu CC, Chen XF, Zhang YW, Xu H, Bu G. Opposing effects of viral mediated brain expression of apolipoprotein E2 (apoE2) and apoE4 on apoE lipidation and $\mathrm{A} \beta$ metabolism in apoE4targeted replacement mice. Mol Neurodegener. 2015;10:6.

23. Zhao L, et al. Intracerebral adeno-associated virus gene delivery of apolipoprotein E2 markedly reduces brain amyloid pathology in Alzheimer's disease mouse models. Neurobiol Aging. 2016;44:159-172.

24. Hudry E, et al. Gene transfer of human Apoe isoforms results in differential modulation of amyloid deposition and neurotoxicity in mouse brain. Sci Transl Med. 2013;5(212):212ra161.

25. Dodart JC, et al. Gene delivery of human apolipoprotein $\mathrm{E}$ alters brain Abeta burden in a mouse model of Alzheimer's disease. Proc Natl Acad Sci U S A. 2005;102(4):1211-1216.

26. Liu CC, et al. ApoE4 accelerates early seeding of amyloid pathology. Neuron. 2017;96(5):1024-1032.e3.

27. Bales KR, et al. Apolipoprotein $\mathrm{E}$ is essential for amyloid deposition in the $\mathrm{APP}(\mathrm{V} 717 \mathrm{~F})$ transgenic mouse model of Alzheimer's disease. Proc Natl Acad Sci U S A. 1999;96(26):15233-15238.

28. Holtzman DM, et al. Apolipoprotein E facilitates neuritic and cerebrovascular plaque formation in an Alzheimer's disease model. Ann Neurol. 2000;47(6):739-747.

29. Huynh TV, et al. Age-dependent effects of apoE reduction using antisense oligonucleotides in a model of $\beta$-amyloidosis. Neuron. 2017;96(5):1013-1023.e4.

30. Kim J, et al. Haploinsufficiency of human APOE reduces amyloid deposition in a mouse model of amyloid- $\beta$ amyloidosis. J Neurosci. 2011;31(49):18007-18012.

31. Bien-Ly N, Gillespie AK, Walker D, Yoon SY, Huang Y. Reducing human apolipoprotein E levels attenuates age-dependent $A \beta$ accumulation in mutant human amyloid precursor protein transgenic mice. J Neurosci. 2012;32(14):4803-4811.

32. Sadowski MJ, et al. Blocking the apolipoprotein E/ amyloid-beta interaction as a potential therapeutic approach for Alzheimer's disease. Proc Natl
Acad Sci U S A. 2006;103(49):18787-18792.

33. Huang Y, Mahley RW. Apolipoprotein E: structure and function in lipid metabolism, neurobiology, and Alzheimer's diseases. Neurobiol Dis. 2014;72 Pt A:3-12.

34. Wahrle SE, et al. Overexpression of ABCA1 reduces amyloid deposition in the PDAPP mouse model of Alzheimer disease. JClin Invest. 2008;118(2):671-682.

35. Wahrle SE, et al. ABCA1 is required for normal central nervous system ApoE levels and for lipidation of astrocyte-secreted apoE. J Biol Chem. 2004;279(39):40987-40993.

36. Riddell DR, et al. The LXR agonist TO901317 selectively lowers hippocampal A $\beta 42$ and improves memory in the Tg2576 mouse model of Alzheimer's disease. Mol Cell Neurosci. 2007;34(4):621-628.

37. Wahrle SE, et al. Deletion of Abca1 increases $\mathrm{A} \beta$ deposition in the PDAPP transgenic mouse model of Alzheimer disease. J Biol Chem. 2005;280(52):43236-43242.

38. Sullivan PM, Mace BE, Maeda N, Schmechel DE. Marked regional differences of brain human apolipoprotein E expression in targeted replacement mice. Neuroscience. 2004;124(4):725-733.

39. Liao F, et al. Effects of CD2-associated protein deficiency on amyloid- $\beta$ in neuroblastoma cells and in an APP transgenic mouse model. Mol Neurodegener. 2015;10:12.

40. Bacskai BJ, Hyman BT. Alzheimer's disease: what multiphoton microscopy teaches us. Neuroscientist. 2002;8(5):386-390. 